\title{
TIPOS DE PERGUNTAS EM ARAWETÉ
}

\section{TYPES OF QUESTIONS IN ARAWETÉ}

\author{
Eliete de Jesus Bararuá Solano \\ Universidade do Estado do Pará, UEPA, Pará, PA, Brasil/Laboratório de Línguas e \\ Literaturas Indígenas, LALLI,Universidade de Brasília
}

Resumo: Neste artigo, apresento os tipos de perguntas em Araweté, uma língua nativa do Brasil classificada por Rodrigues (1985) como pertencente ao Subconjunto V da família linguística Tupí-Guaraní (que é uma das dez famílias que compôem o tronco Tupí), junto com o Ararandewára-Amanajé, Anambé do Cairarí e o Asuriní do Xingu (RODRIGUES e CABRAL, 2002). Por meio da apresentação dessa tipologia referente às perguntas, demonstro que a língua Araweté distingue dois principais tipos, perguntas sim/não ou polares e perguntas informacionais. $\mathrm{O}$ estudo fundamenta-se, principalmente, nas pesquisas e estudos descritivos e histórico-comparativos do Prof. Aryon Rodrigues, de quem fui aluna, tanto na Universidade Federal do Pará (UFPA) quanto na Universidade de Brasília (UnB), e nos trabalhos descritivos e histórico-comparativos da Profa. Ana Suelly Cabral, de quem fui orientanda de mestrado e doutorado e com quem compartilhei (e compartilho) toda a pesquisa descritiva de minha tese sobre a Língua Araweté, da qual este artigo é apenas um dos capítulos.

Palavras-chave: Família Linguística Tupí-Guaraní; Araweté; Perguntas

Abstract: In this paper I present the types of questions in Araweté, a native language of Brasil, which has been classified by Rodrigues (1985) as belonging to Sub-branch V, TupíGuaraní family, Tupian stock, together with Ararandewára, Amanajé, Anambé do Cairarí and Asuriní do Xingu languages (RODRIGUES \& CABRAL, 2002). I demonstrate that Araweté distinguishes two major types of questions: yes/no or polar questions and informational questions. This study is based on Rodrigues descriptive and historical comparative studies.

Keywords: Tupí-Guaraní Family; Araweté; Questions.

\section{Introduçáo}

Neste artigo, apresento uma versão modificada de um dos capítulos de minha tese, intitulada Descrição Gramatical da Língua Araweté, defendida em 2009, na Universidade de Brasília (UnB). Os fundamentos teóricos deste artigo, assim como dos demais estudos descritivos e histórico-comparativos dos quais participo, alicerçam-se nas ricas liçôes do Prof. Aryon Rodrigues, de quem fui aluna, tanto na Universidade Federal do Pará (UFPA) quanto na Universidade de Brasília (UnB), e com quem convivi por quase seis anos no Laboratório de Línguas e Literaturas Indígenas (LALLI), e da Profa. Ana 
Suelly Cabral, de quem fui orientanda de mestrado e doutorado. Foram essas liçóes que me fizeram compreender, no âmbito dos estudos descritivos e histórico-comparativos que, quando se trata de tipos de perguntas em Araweté, língua ainda pouco conhecida, essa língua distingue dois principais tipos de perguntas, perguntas sim/não ou polares e perguntas informacionais, bem como se utiliza de outras construçóes usadas retoricamente para obter respostas.

\section{A partícula $p a$}

Perguntas polares e perguntas informacionais exigem que o constituinte questionado seja seguido da partícula $\boldsymbol{p} \boldsymbol{a}$ - também pronunciada $\boldsymbol{p} \boldsymbol{u}$ quando precede a partícula de foco $k u$ - que expressa 'modalidade alética', marcando o desconhecimento do falante sobre a verdade do conteúdo informacional .

\subsection{Perguntas polares}

Nas perguntas polares, o falante quer apenas saber a verdade ou falsidade do conteúdo da informaçấo questionada:

$\begin{array}{llll}\text { ne } & \text { r-upehi } & \text { pa } & \text { ne } \\ 2 & \mathrm{R}^{\mathrm{I}} \text {-sono } & \mathrm{p}^{1} & 2 \\ \text { 'você está com sono?? }\end{array}$

$$
\begin{array}{lllll}
p \tilde{e} & p u & k u & m e j u & p e-? u \\
23 & \mathrm{p} & \text { FOC } & \text { beiju } & 23 \text {-comer }
\end{array}
$$

'foram vocês que comeram beiju?'

$\begin{array}{llllll}\text { 03) ne } & p u & k u & \text { miniju } & \text { ere-puwĩ } \\ 2 & \mathrm{p} & \mathrm{FOC} & \text { algodão } & \text { 2-fiar } \\ & \text { 'foi você que fiou algodão?' }\end{array}$

\footnotetext{
${ }^{1}$ Abreviaturas: AT OUTRO $=$ atestado pelo outro; $\mathrm{CA}=$ caso alativo; $\mathrm{CR}=$ caso relativo; $\mathrm{CD}$ = caso direcional $; \quad \mathrm{CI}=$ caso inessivo $; \mathrm{DESI}=$ desiderativo $\mathrm{I} ; \mathrm{FOC}=$ foco, $\mathrm{INT}=$ intencional; LOC = localivo; $\mathrm{LP}=$ locativo pontual; $\mathrm{NEG}=$ negação; $\mathrm{NP}=$ nominalizador de predicado; $\mathrm{P}=$ pergunta; $\mathrm{PERM}=$ permissivo; $\mathrm{PL}=$ plural $\mathrm{PROB}=$ probabilidade REIT $=$ reiterativo REF $=$ reflexivo; SUP = suposto; TOP = tópico; 1 = primeira pessoa do singular ; 2 = segunda pessoa do singular; 3 = terceira pessoa do singular; $\mathrm{R}^{1}=$ relacional de contiguidade; $\mathrm{R}^{2}=$ relacional de não-contiguidade; $\mathrm{R}^{4}=$ relacional genérico e humano. 23 = segunda pessoa do plural.
} 
04) $\tilde{p e} \quad p u \quad k u \quad p \dot{\mathbf{x}} d a \quad p e-h i$

$23 \mathrm{p}$ FOC peixe 23-assar

'foram vocês que assaram peixe?'

\subsection{Perguntas informacionais}

Nas perguntas informacionais, o falante busca respostas informativas, evidenciando que as desconhece, como mostram os seguintes exemplos:

05)

$$
\begin{array}{lll}
\text { awa } & \text { pa } & \text { h-uiru-me?e } \\
\text { quem } & \mathrm{P} & \mathrm{R}^{2} \text {-saudade-NP } \\
\text { 'quem está com } & \text { saudade?' }
\end{array}
$$

06) me?e pa rupe

o que $P$ isso

'o que é isso?'

07) awa pa tata u-meni

o que $P$ fogo 3-acender

'quem acendeu o fogo?'

08

$\begin{array}{lccl}\text { awa } & p u & k u & \text { u-wahẽ } \\ \text { o que } & \mathrm{P} & \text { FOC } & \text { 3-chegar } \\ \text { 'quem foi que chegou?' } & \end{array}$

09

$\begin{array}{llllll}\text { awa } & \text { pu } & k u & \text { u-ha } & \text { Jere?ehi } & r \text {-ewe } \\ \text { o que } & \mathrm{P} & \text { FOC } & \text { 3-ir } & \text { Jere?ehi } & \mathrm{R}^{\mathrm{I}} \text {-CA } \\ \text { 'quem } & \text { foi } & & \end{array}$

\subsection{O que pode ser questionado em perguntas polares}

Os constituintes sintáticos que podem ser questionados em perguntas polares são os que funcionam como argumentos, quantificadores numerais, predicados e expressóes adverbiais:

Argumentos

- Sujeito

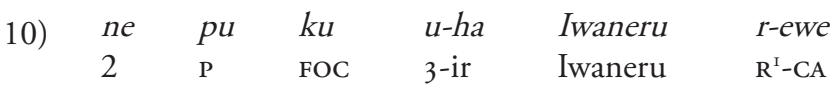
'você foi com o Iwaneru?' 
11) pe $p u \quad k u$ madira pe-ati

$23 \mathrm{P}$ FOC mandioca 23-plantar

'foram vocês que plantaram mandioca?'

12) ne pu ku natã ere-ka $2 \mathrm{p}$ FOC coco.de.babaçu 2-quebrar 'foi você que quebrou coquinho?'

- Predicados verbais

13) pe-ha pu ku pẽ pe-jahu parani-we 23-ir $P$ FOC 23 23-banhar rio-LP 'vocês vão banhar no rio?'

14) ere-karu puta pa ne 2-comer DESI P 2 'você quer comer?'

15) pe-pirĩ puta pa pẽ 2-costurar DESI $P \quad 23$ 'vocês querem costurar?'

-Predicados nominais

16) i-jahu pa ruw̃ niha $\mathrm{R}^{2}$-nova $\mathrm{P}$ esta rede

'é nova esta rede?'

17) pe r-upehi pa pẽ 23 R $\mathrm{R}^{\mathrm{I}}$-sono $\mathrm{P} \quad 2$ 'vocês estão com sono?'

18) ne r-upehi puta pa ne $\begin{array}{lllll} & \mathrm{R}^{\mathrm{I}} \text {-sono } & \text { DESI } & \mathrm{P} & 2\end{array}$ 'você quer dormir?'

-Sintagmas posposicionais

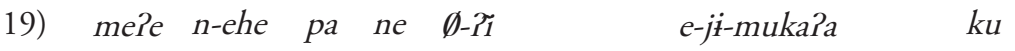

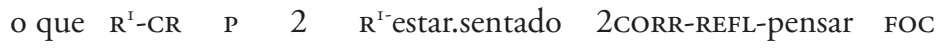
'com o que você está pensando?' 
20)

$\begin{array}{lllll}e & \text { r-eka-ti } & \text { pa } & \text { mide } & \emptyset \text {-ha } \\ \text { esse } & \mathrm{R}^{\mathrm{I}} \text {-estar.em.mov- LP } & \mathrm{P} & 123 & \mathrm{R}^{\mathrm{I}} \text {-ir } \\ \text { 'vamos } & \text { para este lado?' } & & & \end{array}$

$\begin{array}{llllll}\text { 21) } & \text { awa-pi } & \text { pa } & \text { Wi } & n \text { - } u \dot{t} & \text { u-jija } \\ \text { quem-LA } & \mathrm{P} & \text { esses } & \mathrm{R}^{\mathrm{I}-} \text { estar/ficar } & \text { 3-cantar } \\ \text { 'para quem eles estấo cantando?' } & \end{array}$

\subsection{Perguntas informacionais}

Perguntas informacionais são feitas por meio de palavras ou expressóes interrogativas seguidas da partícula alética $\boldsymbol{p a}$. Nas perguntas informacionais, questiona-se sobre o sujeito, o objeto direto, o objeto indireto e demais sintagmas circunstanciais que expressam modo, causa, finalidade, quantidade, que caracteriza a realização de uma ação, de um evento ou de um estado.

\section{$-a w a$}

Por meio da palavra awa, pergunta-se sobre argumentos humanos:

22)

$$
\begin{array}{llll}
\text { awa } & \text { pa } & \text { h-uirũ } & \text { nete-me?e } \\
\text { quem } & \mathrm{P} & \mathrm{R}^{2} \text {-saudade } & \text { muito } \\
\text { 'quem } & \text { está com } & \text { muita saudade dele?' }
\end{array}
$$

23) awa pa tfi-me?e

quem $\mathrm{P}$ bonita-NP

'quem é bonita?'

24) awa pa u-ha ha?iwe

quem $\quad \mathrm{P} \quad 3$-ir amanhã

'quem vai amanhâ?'

$\begin{array}{llll}\text { 25) awa } & \text { pa } & \text { u-purahẽ } & \text { ka?arume } \\ \text { quem } & \mathrm{P} & \text { 3-dançar } & \text { ontem }\end{array}$

'quem dançou ontem?'

26) awa pa u-ha pidanařt

quem $P$ 3-ir mariscar

'quem vai mariscar/pegar peixe?' 
27)

$\begin{array}{lcccl}\text { awa } & \text { pa } & \text { me?e } & \text { u-mupipu } & \text { kumete } \\ \text { quem } & \text { P } & \text { coisa/caça } & \text { 3-cozinhar } & \text { hoje } \\ \text { 'quem é o que vai cozinhar caça hoje? } & \end{array}$

\section{- awa nabu......rupa/nupa}

A partícula $\boldsymbol{n} \boldsymbol{a b u}$ 'suposto', seguindo a palavra awa, contribui com a ideia de 'suposta pessoa', e a palavra rupa - nupa 'probabilidade', no final da sentença, contribui para sinalizar a expectativa do falante em relação à certeza ou falsidade do conteúdo informacional:

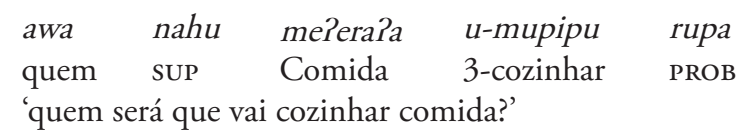

$\begin{array}{llll}\text { awa nahu Mutu u-muje?̃e } & \text { rupa } \\ \text { quem sup } & \text { Motor 3-ligar } & \text { PROB } \\ \text { 'quem será que ligará o motor?' } & \end{array}$

30) awa nahu hemediw $\emptyset$-?u rupa quem SUP remédio $\mathrm{R}^{\mathrm{I}}$-tomar 'quem será que vai tomar remédio?'

$-m e ? e$ não humanos:

A palavra me?e significa 'o que', sendo usado com referência a seres

31) me?e pa rupe

o que $P$ isso

'o que é isso?'

32) me?e pu $\mathrm{ku}$ mide $u-? \mathrm{lu}$ kumete $\begin{array}{lllll}\text { o que } P & \text { FOC } 123 \text {-comer hoje }\end{array}$

'o que nós comeremos hoje?'

33) me?e r-ehe pa ne d-iha

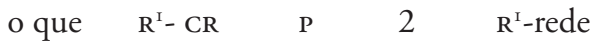

'com o que é (feita) tua rede? 
34) me?e r-ehe he pa pẽ-nã

o que $\mathrm{R}^{\mathrm{I}}$-CR DESII $\mathrm{P}$ 23-ir

'com que vocês desejam ir?'

35) me?e r-ehe pa ne $2 \tilde{i}$

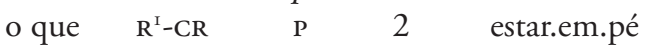

'o que você quer?'

36) me?e r-ahe wi h-eru rupa re?e

o que $\mathrm{R}^{\mathrm{I}}$-CR $3 \quad \mathrm{R}^{2}$-trouxe $\mathrm{PROB} \quad$ AT.OUTRO

'com o que será que a trouxeram?'

\section{- mari}

A palavra mari pode ser traduzida por 'o que' em perguntas como 'o que você têm?' ou 'como' em perguntas 'como você fez tal coisa?':

37) mari pu ku ne iwi-ku-karu

o que $\mathrm{P}$ FOC 2 terra-buraco-cavar

'como você cavou.buraco?

38) mari pa ne re?e

o que $\mathrm{P} \quad 2$ AT.OUTRO

'o que você tem?'

39) mar̃ pa re?e

o que $\mathrm{P}$ AT.OUTRO

'o que ele tem?'

40) mari $p u \quad k u \quad p \tilde{e}$

o que $P$ FOC 23

'o que vocês têm?'

-marima $\sim$ marïmũ

A palavra marïma $~$ marïmũ requer informações sobre o 'porquê dos fatos:

41) marima $p u \quad k u$ ne $r$-udi ja ka?arume a?t $\begin{array}{llllll}\text { por que } & \mathrm{P} & \text { FOC } & 2 & \mathrm{R}^{\mathrm{T}} \text {-voltar } & \text { NEG ontem REIT }\end{array}$ 'por que você não voltou ontem?'

42) marima $\mathrm{pu} \mathrm{ku}$ ne maka Ø-mere ja we ha he r-e por que $\mathrm{P}$ FOC 2 manga $\mathrm{R}^{\mathrm{I}}$-dar NEG TOP ir $1 \mathrm{R}^{\mathrm{I}}-\mathrm{CR}$ 'por que você não vai dar manga para mim?' 
43) marimũ pa ne r-uri a?i $\begin{array}{lllll}\text { por que } & P & 2 & R^{\mathrm{I}} \text {-alegre } \quad \text { REIT }\end{array}$ 'por que você está alegre?'

44) marimũ pa ne $\emptyset$-ha belem-ipi por que $\quad \mathrm{P} \quad 2 \quad \mathrm{R}^{\mathrm{I}}$-ir belem-LA 'por que você vai para Belém?

45) marimũ pa u-wahẽ ja nu porque $\mathrm{P}$ 3-chegar NEG de novo 'por que ele não chegou de novo?

45) marimũ pa ku i-ji-pihi ja we re?e por que $\mathrm{P}$ FOC $\mathrm{R}^{2}$-REFL-pintar NEG TOP AT.OUTRO 'por que ela não se pintou?

- mamu

A palavra mamu é usada quando se quer perguntar sobre 'o lugar para onde se foi ou de onde se veio':

46) mamu pu ku ne $\emptyset$-ja $\begin{array}{lllll}\text { onde } \quad \mathrm{P} & \text { FOC } & 2 & \mathrm{R}^{\mathrm{I}} \text {-vir }\end{array}$ 'onde você veio?'

47) mamu pu ku u-ja onde $\quad \mathrm{P} \quad$ FOC 3 -vir 'onde ele veio?'

48) таmu pu ku pe Ø-ha onde $\quad \mathrm{P} \quad$ FOC $23 \quad \mathrm{R}^{\mathrm{I}}$-ir 'onde vocês foram?'

\section{$-\boldsymbol{m} \tilde{\boldsymbol{i}} \sim \boldsymbol{m} \tilde{i} h \tilde{i}$}

A palavra interrogativa $\boldsymbol{m} \tilde{\boldsymbol{i}} \sim \boldsymbol{m} \tilde{\boldsymbol{i}} \boldsymbol{h} \tilde{\mathbf{i}}$ é usada quando se pergunta sobre um lugar (estático) em que algo ou alguém se encontra:

49) mi pa Kunirikuti e?e onde $\mathrm{P}$ Kunirikuti esse 'cadê a Kunirikuti?' 'onde está a Kunirikuti?'

50) mĩ pa Tatuaru e?e onde $\mathrm{P}$ Tatuaru esse 'cadê o Tatuaru?' 
51) mĩhĩ pa ja e?e

onde $\mathrm{P}$ dono/senhor esse

'cadê o dono?'

52) mĩhĩ pa jarut $\int u \quad e ? e$

onde $P$ canoa esse

'cadê a canoa?

53) mĩhĩ a-ka ime pu ku Nivaldo u-ha re?e

onde I-estar/ficar quando/se $\mathrm{P}$ FOC Nivaldo 3-ir AT.OUTRO 'onde eu estava quando o Nivaldo foi?'

54) mĩhĩ a-ka ime pu ku ja $\tilde{I}$ re?e onde I-estar/ficar quando/se P FOC onça estar.em.pé AT.OUTRO 'onde eu estava quando a onça apareceu?

\section{- mïhijije mijije}

A palavra mîhijije $\sim$ mĩjije é usada para perguntar sobre o 'quando' ou 'quanto', com respeito ao acontecimento dos fatos. Essa palavra ocorre no início de oraçóes com predicados modificados pela conjunção $\boldsymbol{m e} \boldsymbol{e}$, a qual pode ser opcionalmente seguida pela partícula nupa:

55) mîhĩjije pa Nivaldo r-udi me

Quando $\mathrm{P}$ Nivaldo $\mathrm{R}^{\mathrm{I}}$-voltar quando/se 'quando será que o Nivaldo voltará?'

56) mĩhĩjije pa Silvana Ø-wahẽ me Quando $P$ Silvana $\mathrm{R}^{\mathrm{I}}$-chegar quando/se 'quando será que a Silvana chegará?'

57) mĩhijije pa Juliana r-udi me nupa Quando $\mathrm{P}$ Juliana $\mathrm{R}^{\mathrm{I}}$-voltar quando/se $\mathrm{PROB}$ 'quando será que a Juliana vai voltar?'

58) mĩhĩjije pa amĩ $\emptyset$-ja me nupa Quando $\mathrm{P}$ chuva $\mathrm{R}^{\mathrm{I}}$-vir quando/se PROB 'quando será que a chuva virá?'

59) mĩhijije pa Benigno Ø-ja me nupa Quando $\mathrm{P}$ Benigno $\mathrm{R}^{\mathrm{I}}$-vir quando/se $\mathrm{PROB}$ 'quando será que o Benigno virá?' 


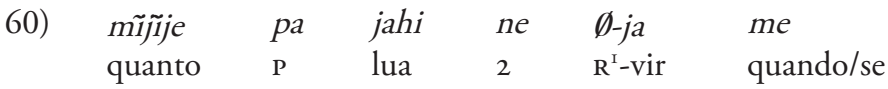
'quantas luas faltam para a tua vinda?'

$\begin{array}{lllll}\text { 61) mijjije } & \text { pa } & \text { pida } & n \text {-eru } & r e ? u \\ \text { quanto } & \mathrm{P} & \text { peixe } & \mathrm{R}^{\mathrm{I}} \text {-trazer } & \text { esse }\end{array}$ 'quantos peixes ele trouxe?'

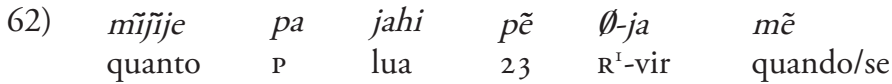
'quantas luas faltam para vocês virem?'

\section{Sintagmas adverbiais complexos usados para perguntar}

Finalmente, palavras interrogativas, combinadas com morfologia casual ou com posposiçôes, são usadas para perguntar sobre circunstâncias diversas:

- na direçáo de onde

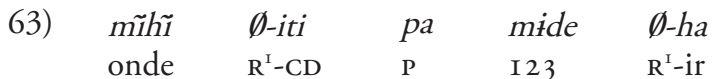
'para que lado nós iremos?'

64) mîhi $\emptyset$-iti $\quad p u \quad k u \quad u$-iwũ onde $\quad \mathrm{R}^{\mathrm{I}-\mathrm{CD}} \quad \mathrm{P} \quad$ FOC 3 -flechar 'em que direção ele flechou?'

- instrumento

65) me?e Ø-iwe pu ku madipa e-kiti a?i o que $\mathrm{R}^{\mathrm{I}}$-CI $\mathrm{P}$ FOC mandioca 2-ralar REIT 'com o que rala mandioca?'

66) me?e $\emptyset$-iwe pu ku pẽ $\emptyset$-madira $\begin{array}{llllll}\text { o que } & \mathrm{R}^{\mathrm{I}} \text {-CI } & \mathrm{P} & \text { FOC } & 23 & \mathrm{R}^{\mathrm{I}} \text {-mandioca }\end{array}$ 'com o que rala a mandioca de vocês?' 
- em que lugar

67) $\begin{array}{llllll}\text { mî-we } & \text { pu } & k u & \text { ut } \tilde{i} & \text { u-mara } & \text { h-akawa } \\ \text { onde-LP } & \text { P } & \text { FOC } & \text { farinha } & \text { 3-mandar } & \mathrm{R}^{2} \text {-jogar }\end{array}$ 'onde ele mandou jogar a farinha?'

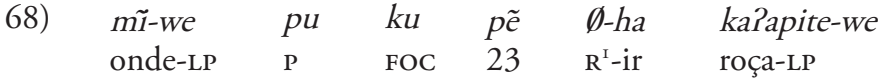
'onde vocês vão na roça?'

\section{-em que direção}

69) Awa Ø-kati pu ku i-?i

o que $\quad \mathrm{R}^{\mathrm{I}}$-LD $\quad \mathrm{P} \quad$ FOC $\quad \mathrm{R}^{2}$-estar.em.pé

'em direção de quem ele está?'

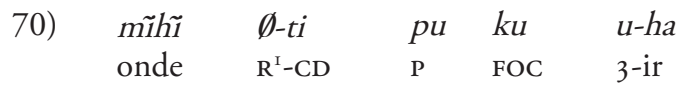

'em direção de quem ele foi?'

71) Awa $\emptyset$-kati pu ku h-a?a u-ita o que $\quad \mathrm{R}^{\mathrm{I}}$-LD $\quad \mathrm{P} \quad$ FOC $\quad \mathrm{R}^{2}$-atravessar 3-nadar 'em direção de quem ele nadou?'

- a respeito de que

72) meie r-ehe pa ne ku ere-mukara ku $\begin{array}{llllll}\text { o que } & \mathrm{R}^{\mathrm{I}} \text {-com } & \mathrm{P} & 2 & \text { FOC } & \text { 2-pensar }\end{array}$ 'em que você está pensando?'

73) me?e r-ehe pa rupu ku u-muka?a ku o que $\mathrm{R}^{\mathrm{I}}$-com $\mathrm{P}$ este.deitado FOC 3-pensar FOC 'em que ela está pensando?'

74) me?e r-ehe pa $t$-u u-ji-mukara $k u$ o que $\quad \mathrm{R}^{\mathrm{I}}$-com $\mathrm{P} \quad \mathrm{R}^{2}$-pai 3-REFL-pensar FOC 'em que ele se lembra do pai dele?

75) mepe r-e pa mide r-ui u-ji-muka?a ku o que $\mathrm{R}^{\mathrm{I}}$-com $\mathrm{P}$ I23 $\mathrm{R}^{\mathrm{I}}$-estar/ficar 3-REFL-pensar FOC 'em que nós estamos pensando/lembrando?' 

76) mere r-ehe pa p̃e r-ui pe-ji-mukara ku o que $\mathrm{R}^{\mathrm{I}}$-com $\mathrm{P} \quad 23 \quad \mathrm{R}^{\mathrm{I}}$-estar/ficar 23-REF-pensar FOC 'em que vocês estáo pensando/lembrando?'

\subsection{Perguntas retóricas}

Os Araweté fazem também uso de orações sem a partícula alética, para obter uma confirmação ou uma negação relativa ao conteúdo informacional expresso, como por exemplo, quando dizem:

$$
\begin{aligned}
& \text { ne } \quad r-u \\
& 2 \quad \mathrm{R}^{\mathrm{I}} \text {-pai } \\
& \text { 'teu pai?' ou 'você tem pai?' }
\end{aligned}
$$

78) ne r-erekũ

$2 \mathrm{R}^{\mathrm{I}}$-marido

'teu marido?' ou 'você tem marido?'

79) $p \tilde{e} \quad \emptyset$-tfirima

$23 \mathrm{R}^{\mathrm{I}}$-cansado

'estáo cansados?' ou 'vocês tem cansaço?'

Quando um Araweté se aproxima pela primeira vez de alguém, pode proferir esse enunciado para obter uma resposta sim/não. Há ocasióes em que conhece a resposta, mas usa a forma declarativa como pergunta retórica.

\section{Conclusáo}

Neste artigo, descrevemos os diferentes tipos de perguntas identificadas até o presente na língua Araweté. Mostramos que perguntas polares e perguntas informacionais exigem que o constituinte questionado seja seguido da partícula $p a$, que é uma das expressóes de modalidade 'alética' nessa língua. Apresentamos vários exemplos, os quais contêm as diferentes partículas modalizadoras de perguntas e ressaltamos que uma oração declarativa pode ser usada retoricamente para fazer perguntas.

A variedade de partículas usadas em perguntas na língua Araweté corresponde às distinçôes culturais que fazem os Araweté, como as de estático versus dinâmico, com respeito ao conceito de onde, ou a não distinção de quanto versus quando. 
Finalmente, os exemplos apresentados mostram que a posição periférica à esquerda da sentença é a posição dos constituintes questionados, ou seja, é uma posição de tópico/foco.

\section{Referências}

CABRAL, Ana Suelly A.; SOLANO, Eliete de Jesus B. Sobre as línguas TupíGuaraní do Xingu e os seus deslocamentos pré-históricos. In: SIMÓES, Maria do Socorro (org). Sob o signo do Xingu. Belém: UFPA/IFNOPAP, 2003, p. 17-36.

CASTRO, Eduardo B. Viveiros de. Araweté: os deuses canibais. Rio de Janeiro: Jorge Zahar Ed., 1986.

LEITE, Yonne F; VIERA, Márcia D. Observações Preliminares sobre a Língua Araweté. Moara - Revista dos Cursos de Pós-Grad. em Letras da UFPA, n.9, p. 7-31. Belém, 1998.

MAGAlHÃES, J. V. Couto de. O Selvagem. Edição comemorativa do centenário da $1^{a}$ edição. São Paulo: Itatiaia, [1876]1975.

RODRIGUES, Aryon D. Relaçóes internas na família lingüística TupíGuaraní. Revista de Antropologia, n. 27/28, p. 33-53. Săo Paulo, 1985.

Línguas brasileiras: para o conhecimento das línguas indígenas. São Paulo: Loyola, 1986.

; CABRAL, Ana Suelly A. C. Revendo a classificaçáa interna da família Tupí-Guaraní. In: Ana Suelly A. C. Cabral \& Aryon D. Rodrigues (Org.). Línguas indígenas brasileiras: fonologia, gramática, história. vol. I:327-337. Belém: EDUFPA, 2002.

SOLANO, Eliete de Jesus Bararuá. A Posiçáo do Araweté na Família Linguística Tupi-Guarani: considerações linguísticas e históricas. Dissertação (Mestrado em Letras) - Centro de Letras e Artes da Universidade Federal do Estado do Pará, Belém, 2004.

; Eliete de Jesus Bararuá; CABRAL, Ana Suelly A C. Nós Somos Pele: o desenvolvimento de um pronome "nós" em Araweté. In: Workshop Linguistica Histórica e línguas em contato - Línguas Indígenas do Brasil e de áreas adjacentes, Brasília: UnB, 2005 
; Eliete de Jesus Bararuá. Descriçáo Gramatical da Língua Araweté. Tese (Doutorado em Linguística) - Universidade de Brasília, Instituto de Letras, Brasília, 2009.

Tipos de perguntas em Araweté Eliete de Jesus Bararuá Solano Recebido em 21/06/2015 Aprovado em 30/09/2015 